\title{
Effectiveness of a multidisciplinary team for nutrition support in a trauma intensive care unit
}

\author{
Eunsuk Oh ${ }^{1,2}$, Hongjin Shim ${ }^{1,3,4}$, Hyon Ju Yon ${ }^{1,5}$, Jin Sil Moon ${ }^{6}$, Dae Ryong Kang ${ }^{6}$, Ji Young Jang ${ }^{7}$ \\ ${ }^{1}$ Nutrition Support Team and ${ }^{2}$ Dapartment of Pharmacy, Wonju Severance Christian Hospital, Wonju, ${ }^{3}$ Department of Surgery, Yonsei University \\ Wonju College of Medicine, Wonju; ${ }^{4}$ Trauma Center and ${ }^{5}$ Department of Nutrition Services, Wonju Severance Christian Hospital, Wonju; ${ }^{6}$ Department of \\ Biostatistics, Yonsei University Wonju College of Medicine, Wonju, 'Department of Surgery, National Health Insurance Service Ilsan Hospital, Goyang, Korea
}

Background: We evaluated clinical and nutritional outcomes according to multidisciplinary team involvement in nutrition support in a regional trauma intensive care unit (TICU).

Methods: We retrospectively compared the outcomes for 339 patients admitted to the TICU for $>5$ days depending on nutrition support team (NST) involvement $(n=176)$ and non-NST involvement $(n=163)$.

Results: The mean age and injury severity score (ISS) were $57.3 \pm 16.7$ years and $18.6 \pm 9.7$, respectively. Fifty-three patients (15.6\%) had shock on admission and 182 (53.7\%) underwent surgery during TICU admission. Some patients were admitted to neurosurgery (46\%), general surgery (35.4\%), and other (18.6\%) departments. There were significant differences in the ISS, Acute Physiology and Chronic Health Evaluation (APACHE) II score, shock on TICU admission, and initial laboratory results. After propensity score matching, the total delivered/required caloric ratio and total delivered/required protein ratio were significantly higher in the NST group than in the non-NST group (calorie: $80.4 \%$ vs. $66.7 \%, P=0.007$; protein: $93.1 \%$ vs. $68.3 \%, \mathrm{P}<0.001)$. The NST group had an adequate protein supply more frequently than the non-NST group (protein: $48.0 \%$ vs. $25.8 \%, P=0.002$ ). There was no significant difference in survival, even after adjustment for risk factors using Cox proportional hazard analysis.

Conclusions: The results of our study suggest that multidisciplinary team involvement in nutrition support in TICU patients may improve nutritional, but not clinical, outcomes.

Key Words: injuries; intensive care unit; multidisciplinary team; nutrition support

\section{INTRODUCTION}

Malnutrition is identified in approximately $30 \%-55 \%$ of hospitalized patients, and nutritional status is closely related to clinical outcomes for patients [1-3]. In multiple studies, malnutrition has been reported as an important risk factor for mortality and morbidity in critically ill patients [4,5]. Therefore, the National Health Insurance (NHI) of Korea established provisions for the reimbursement of multidisciplinary teams for nutrition support in critically ill patients in 2015, and nutrition support teams (NSTs) now conduct nutritional assessments and nutrition support for patients in each hospital [6]. It has also been reported that nutrition support reduces mortality and the incidences of infectious complications in trauma patients [7-10]. However, a recent multi-center study showed that adequate support of energy and protein

\section{Original Article}

Received: May 26, 2020

Revised: July 22, 2020

Accepted: July 29, 2020

Corresponding author

Ji Young Jang

Department of Surgery, National

Health Insurance Service Ilsan

Hospital, 100 Ilsan-ro, Ilsandong-gu,

Goyang 10444, Korea

Tel: +82-31-900-0975

Fax: +82-31-900-0138

E-mail: drjangjiyoung@gmail.com

Copyright $@ 2020$ The Korean Society of Critical Care Medicine

This is an Open Access article distributed under the terms of Creative Attributions Non-Commercial License (https:// creativecommons.org/li-censes/by-nc/4.0/) which permits unrestricted noncommercial use, distribution, and reproduction in any medium, provided the original work is properly cited. 
was provided for only $50 \%-60 \%$ of trauma patients [11]. Seventeen regional trauma centers were announced by the Korean government in 2012. Currently, 11 trauma centers have been opened and supported by the Korean Ministry of Health and Welfare, with each center containing at least 20 beds in the trauma intensive care units (TICUs) [12]. However, since the NST project by the NHI in Korean trauma centers was just recently introduced and its recommendations were not mandatory for the attending physicians, the effectiveness of multidisciplinary teams for NST is still unclear. In addition, literature regarding nutritional issues in injured patients admitted to the TICU is limited. Therefore, the purpose of this study was to evaluate clinical and nutritional outcomes according to involvement of a multidisciplinary team for nutrition support in injured patients admitted to the TICU.

\section{MATERIALS AND METHODS}

\section{Patient Selection and Data Collection}

In this observational study, the medical records of injured patients (ages > 18 years) who were admitted to the TICU of a regional trauma center in a tertiary university hospital from January 2015 to December 2015 were analyzed retrospectively. The design of this study was approved by the Institutional Review Board of Wonju Severance Christian Hospital (IRB No. CR318059), and the need for informed consent was waived due to the retrospective nature of the study. For evaluation of patients' nutritional outcomes without the effects of early death or transfer to another hospital, patients who were discharged or transferred within 5 days were excluded from our study.

The clinical characteristic data collected included age, sex, injury severity score (ISS), Acute Physiology and Chronic Health Evaluation (APACHE) II score on TICU admission, occurrence of shock at the time of TICU admission, surgery during TICU admission, and admission department. Nutritional information, such as weight, height, body mass index, timing of nutrition initiation, initial nutritional route (parenteral nutrition [PN], tube feeding, oral, or mixed: enteral nutrition [EN] and $\mathrm{PN})$, calculated energy requirement (kcal/day), protein requirement (g/day), and consultation to NST, was also identified. Energy requirements were calculated using either a simplistic formula (25-30 kcal/ $\mathrm{kg}$ ) or the Harris-Benedict equation. Adequate amounts of calories and protein were defined as achievement of $70 \%-110 \%$ of the calculated amounts during TICU admission.

\section{KEY MESSAGES}

- Nutrition support team in critically ill injured patients improved nutritional, but not clinical, outcomes.

- In order to solve these problems, it is necessary to ensure continuity in nutrition support for patients by organizing the nutrition support team round with the multidisciplinary round in the intensive care unit.

\section{Outcome Evaluations}

The primary study end-point was nutritional outcomes, which included total delivered/required caloric ratio (\%) and total delivered/required protein ratio (\%) during TICU admission, and the rate of adequate calorie and protein supply during TICU admission. The secondary end-point was clinical outcomes, which were duration of TICU stay, duration of hospitalization, ICU mortality rate, and mortality rate.

\section{TICU System}

The 20-bed TICU in this study was a semi-closed type. In general, injured patients were admitted to each department (department of trauma surgery, neurosurgery, orthopedic surgery, and cardiothoracic surgery) depending on their main injury site, and patients were only referred to a surgical intensivist if the physician in charge decided that a transfer was necessary.

\section{Nutrition Support Team}

Patients were indicated for the NST program if they had hypoalbuminemia $(<3.0 \mathrm{~g} / \mathrm{dl})$, were on PN or EN, were admitted to the ICU, or if malnutrition was suspected by the physician. Although there were indications as noted above, multidisciplinary consult services were only conducted if the physician in charge determined that NST consult was necessary. An alert in the order communication system was received after 48 hours from TICU admission if NST consultation was required. The NST provided information about energy and protein targets, nutritional route, and other recommendations associated with patient condition at a multidisciplinary round twice a week. The NST in our hospital consisted of a trauma surgeon (surgical intensivist), a clinical nutritionist, a pharmacist, and a ward nurse. The trauma surgeon assessed patient status, and recommended start timing and the route of nutrition. The clinical nutritionist calculated the amount of nutrition required and the recommended EN prescription. The pharmacist monitored issues with prescribed medications and PN use. Acceptance of these recommendations was 
decided by the attending physician. All of the NST members were required to attend educational training and were certified in total nutrition therapy for critical professionals.

\section{Statistical Analysis}

Continuous variables were presented as the mean value \pm standard deviation and categorical variables were presented as frequency (percentage). The two groups according to NST consultation were compared using the two-sample t-test, chisquare test, or Fisher's exact test. To reduce bias in patient selection, propensity score matching was performed. Propensity scores were estimated for each patient using multivariate logistic regression analysis. The covariates included in the calculation were age, sex, occurrence of shock at the time of TICU admission, admission department, ISS, APACHE II score, and length of TICU stay. A one to one matched analysis using nearest-neighbor matching with a caliper distance of 0.05 without replacement was performed based on the estimated propensity score of each patient group. Survival curves were constructed using the Kaplan-Meier method for comparison between the curves. Multivariable analysis of outcomes was performed using the Cox proportional hazards regression model. Statistical significance was accepted for two-sided P-values of $<0.05$. Statistical analyses were performed using SAS 9.4 software (SAS Inc., Cary, NC, USA) and IBM SPSS ver. 23.0 (IBM Inc., Armonk, NY, USA).

\section{RESULTS}

\section{Patient Characteristics}

Among 565 injured patients admitted to the TICU, 153 patients who had any missing variable, and 73 patients who died, were discharged, or were transferred to another hospital within 5 days of admission, were excluded from this study. The final enrolled population included 339 patients (Figure 1). The mean age of patients was $57.3 \pm 16.7$ years and there were 267 males (78.8\%). The mean ISS and APACHE II scores were 18.6 \pm 9.7 and $13.3 \pm 6.9$, respectively. Fifty-three patients $(15.6 \%)$ were in shock at the time of TICU admission, and 182 patients (53.7\%) underwent surgery during TICU admission. Nutritional support was initiated $2.4 \pm 1.8$ days after TICU admission. The initial nutrition was $\mathrm{PN}$ in 279 patients (82.3\%), oral route in 44 patients (13.6\%), and tube feeding in five patients (1.5\%). The ICU mortality rate was $8.6 \%$ and the overall mortality rate was 9.4\%. Consultation with the NST occurred for 176 patients (NST group), and 163 patients did not receive NST consultation (non-NST group) (Table 1).

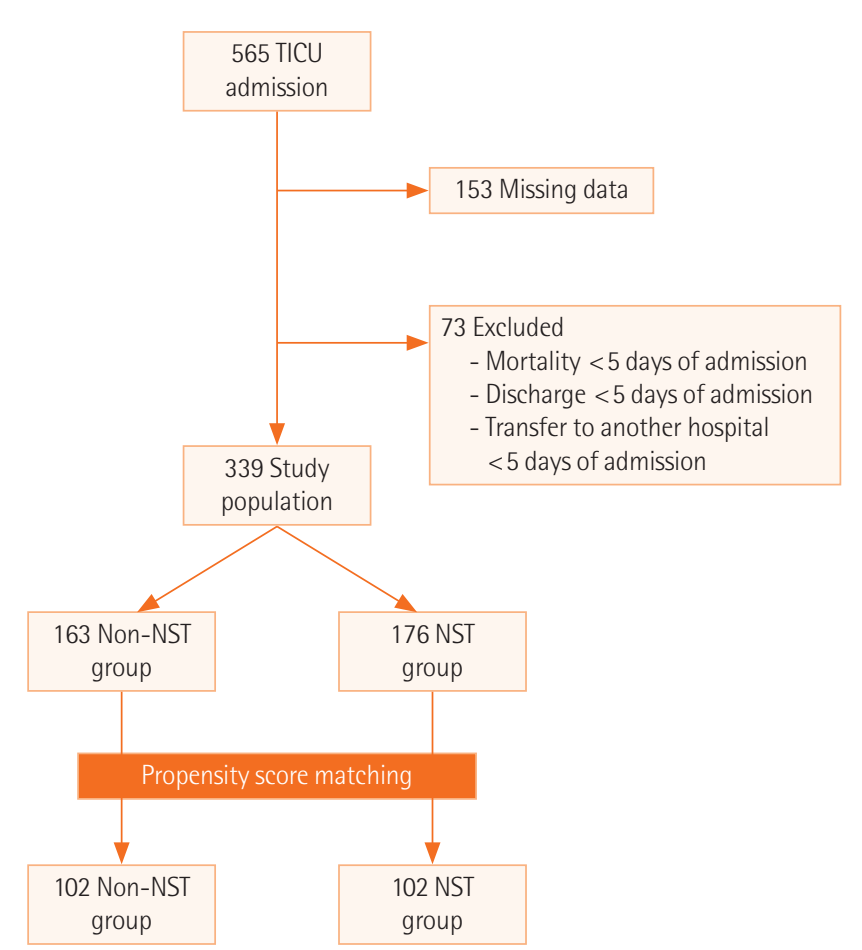

Figure 1. Patient flowchart. TICU: trauma intensive care unit; NST: nutrition support team.

\section{Comparison between Non-NST group and NST Group}

When the non-NST group and NST group were compared, there were significant differences in patient characteristics, including ISS, APACHE II score, occurrence of shock at the time of TICU admission, surgery in the TICU, initial hemoglobin, initial serum albumin, and the initial nutrition route. The ICU mortality rate and overall mortality rate were also significantly higher in the NST group than in the non-NST group (21.5\% vs. $6.3 \%$ and $14.2 \%$ vs. $4.3 \%$, respectively) (Table 1 ).

Nutritional and Clinical Outcomes between the Non-NST Group and NST Group after Propensity Score Matching After propensity score matching, there were no significant differences in the ISS, APACHE II score, the occurrence of shock at the time of TICU admission, surgery in the TICU, initial hemoglobin, and serum albumin (Table 2). The total delivered/ required caloric ratio and total delivered/required protein ratio were significantly higher in the NST group than in the nonNST group (calorie: $80.4 \%$ vs. $66.7 \%, \mathrm{P}=0.007$; protein: $93.1 \%$ vs. $68.3 \%, \mathrm{P}<0.001)$. Adequate protein supply was more common in the NST group than in the non-NST group ( $48.0 \%$ vs. $25.8 \%, \mathrm{P}=0.002$ ) (Table 3). However, there was no significant difference in survival between the 2 groups $(\mathrm{P}=0.740)$ (Figure 2). After adjustment for age, sex, occurrence of shock, admis- 
Table 1. Patient characteristics according to NST consultation before propensity score matching

\begin{tabular}{|c|c|c|c|c|}
\hline Variable & Total $(n=339)$ & Non-NST $(n=163)$ & NST $(n=176)$ & P-value \\
\hline Age (yr) & $57.3 \pm 16.7$ & $56.3 \pm 16.9$ & $58.2 \pm 16.4$ & 0.297 \\
\hline Male sex & 267 (78.8) & $38(23.3)$ & 34 (19.3) & 0.369 \\
\hline ISS & $18.6 \pm 9.7$ & $16.2 \pm 9.1$ & $20.8 \pm 9.6$ & $<0.001$ \\
\hline APACHE II score & $13.3 \pm 6.9$ & $12.0 \pm 6.4$ & $14.6 \pm 7.2$ & $<0.001$ \\
\hline Shock at the time of TICU admission & $53(15.6)$ & $17(10.4)$ & $36(20.5)$ & 0.011 \\
\hline Surgery during TICU admission & $182(53.7)$ & $77(47.2)$ & $105(59.7)$ & 0.022 \\
\hline Initial hemoglobin (g/dl) & $11.9 \pm 2.1$ & $12.3 \pm 2.2$ & $11.6 \pm 1.9$ & 0.002 \\
\hline Initial serum albumin (g/dl) & $3.4 \pm 0.7$ & $3.5 \pm 0.6$ & $3.3 \pm 0.7$ & 0.041 \\
\hline Admission department & & & & 0.098 \\
\hline Neurosurgery & $156(46.0)$ & $67(41.1)$ & $89(50.6)$ & \\
\hline General surgery & $120(35.4)$ & $67(41.1)$ & $53(30.1)$ & \\
\hline Other & $63(18.6)$ & $29(17.8)$ & 34 (19.3) & \\
\hline Weight (kg) & $66.4 \pm 12.0$ & $66.5 \pm 11.8$ & $66.2 \pm 12.2$ & 0.821 \\
\hline Height (cm) & $166.9 \pm 8.8$ & $166.6 \pm 8.8$ & $167.0 \pm 8.9$ & 0.702 \\
\hline BMI $\left(\mathrm{kg} / \mathrm{m}^{2}\right)$ & $23.7 \pm 3.3$ & $23.9 \pm 3.5$ & $23.6 \pm 3.2$ & 0.499 \\
\hline Timing of nutrition start (day) & $2.4 \pm 1.8$ & $2.4 \pm 1.6$ & $2.3 \pm 2.1$ & 0.796 \\
\hline Initial nutrition route & & & & $<0.001$ \\
\hline PN & 279 (82.3) & 120 (73.6) & 159 (90.3) & \\
\hline Oral & 44 (13.6) & $35(21.5)$ & $11(6.3)$ & \\
\hline Tube feeding & $5(1.5)$ & $1(0.6)$ & $4(2.3)$ & \\
\hline Mixed & $11(3.2)$ & $6(3.7)$ & $4(2.3)$ & \\
\hline Calculated calorie requirement (kcal) & $1,516 \pm 202$ & $1,534 \pm 205$ & $1,501 \pm 200$ & 0.143 \\
\hline Calculated protein requirement (g) & $74.5 \pm 11.7$ & $74.5 \pm 10.9$ & $74.6 \pm 12.3$ & 0.897 \\
\hline Total delivered/required caloric ratio in TICU (\%) & $80.4 \pm 38.7$ & $64.1 \pm 36.3$ & $93.8 \pm 35.5$ & $<0.001$ \\
\hline Total delivered/required protein ratio in TICU (\%) & $88.8 \pm 51.0$ & $65.5 \pm 49.5$ & $108.1 \pm 43.7$ & $<0.001$ \\
\hline Adequate energy supply & 118 (36.9) & $39(27.1)$ & 79 (44.9) & 0.001 \\
\hline Adequate protein supply & $102(31.8)$ & $36(24.8)$ & $66(37.5)$ & 0.015 \\
\hline Duration of TICU admission (day) & $10.4 \pm 12.4$ & $5.5 \pm 4.8$ & $14.9 \pm 15.3$ & $<0.001$ \\
\hline Duration of hospitalization (day) & $24(6-86)^{a}$ & $22.6 \pm 15.7$ & $34.5 \pm 20.8$ & $<0.001$ \\
\hline ICU mortality & $29(8.6)$ & $6(6.3)$ & $23(21.5)$ & 0.002 \\
\hline Overall mortality & $32(9.4)$ & $7(4.3)$ & $25(14.2)$ & 0.002 \\
\hline
\end{tabular}

Values are presented as mean \pm standard deviation or number (\%).

NST: nutrition support team; ISS: injury severity score; APACHE: Acute Physiology and Chronic Health Evaluation; TICU: trauma intensive care unit; BMI: body mass index; PN: parenteral nutrition; ICU: intensive care unit.

aedian (range).

sion department, ISS, and APACHE II score by Cox proportional hazards analysis, there was no significant difference in survival between the two groups (Table 4).

\section{DISCUSSION}

After propensity score matching analysis to adjust for ISS and severity at the time of TICU admission between the NST group and the non-NST group, the clinical characteristics of the two groups were similarly corrected. A multidisciplinary approach using a NST increased the nutrition received, including the total delivered/required caloric and protein ratios, and the rate of adequate calorie supply. This change seemed to be due to the fact that the NST provided adequate information about energy and protein targets, and these recommendations were repeatedly mentioned by the multidisciplinary team. Howev- 
er, there was no improvement in the nutrition route or clinical outcomes, such as the mortality rate and length of hospital

Table 2. Patient characteristics according to NST consultation after propensity score matching

\begin{tabular}{|c|c|c|c|}
\hline Variable & $\begin{array}{c}\text { Non-NST } \\
(n=102)\end{array}$ & $\begin{array}{c}\text { NST } \\
(n=102)\end{array}$ & $\begin{array}{c}\mathrm{P}- \\
\text { value }\end{array}$ \\
\hline Age (yr) & $56.3 \pm 17.2$ & $58.3 \pm 16.7$ & 0.395 \\
\hline Male sex & $80(78.4)$ & 79 (77.5) & 0.866 \\
\hline ISS & $19.0 \pm 9.8$ & $20.0 \pm 10.2$ & 0.510 \\
\hline APACHE II score & $12.9 \pm 7.0$ & $12.1 \pm 6.6$ & 0.405 \\
\hline Shock at the time of TICU admission & $13(12.8)$ & $9(8.8)$ & 0.367 \\
\hline Surgery during TICU admission & $54(52.9)$ & $50(49.0)$ & 0.575 \\
\hline Initial hemoglobin (g/dl) & $12.0 \pm 2.2$ & $11.8 \pm 1.8$ & 0.533 \\
\hline Initial albumin & $3.4 \pm 0.7$ & $3.5 \pm 0.7$ & 0.624 \\
\hline Admission department & & & 0.031 \\
\hline Neurosurgery & $45(44.1)$ & $46(44.1)$ & \\
\hline General surgery & $46(45.1)$ & $33(32.4)$ & \\
\hline Others & $11(10.8)$ & $24(23.5)$ & \\
\hline Weight (kg) & $66.7 \pm 12.3$ & $66.9 \pm 12.7$ & 0.874 \\
\hline Height (cm) & $167.3 \pm 8.2$ & $167.1 \pm 9.2$ & 0.861 \\
\hline BMI $\left(\mathrm{kg} / \mathrm{m}^{2}\right)$ & $23.7 \pm 3.6$ & $23.9 \pm 3.4$ & 0.719 \\
\hline Timing of nutrition start (day) & $2.5 \pm 1.7$ & $2.4 \pm 2.1$ & 0.557 \\
\hline Initial nutrition route & & & 0.003 \\
\hline PN & $83(81.4)$ & $90(88.2)$ & \\
\hline Oral & $15(14.7)$ & $9(8.82)$ & \\
\hline Tube feeding & 0 & $2(1.96)$ & \\
\hline Mixed & $4(3.92)$ & $1(0.98)$ & \\
\hline Calculated calorie requirement (kcal) & $1,554 \pm 213$ & $1,515 \pm 212$ & 0.213 \\
\hline Calculated protein requirement (g) & $75.1 \pm 11.1$ & $75.5 \pm 12.7$ & 0.827 \\
\hline
\end{tabular}

Values are presented as mean \pm standard deviation or number (\%).

NST: nutrition support team; ISS: injury severity score; APACHE: Acute Physiology and Chronic Health Evaluation; TICU: trauma intensive care unit; BMI: body mass index; PN: parenteral nutrition. stay. This result may have been due to several reasons. First, the NST was not able to change nutrition orders and could only make recommendations regarding nutritional issues, because the NST did not have authority to prescribe. Compliance with recommendations made by the NST had an impact on clinical outcomes. Second, it is difficult to conduct NST

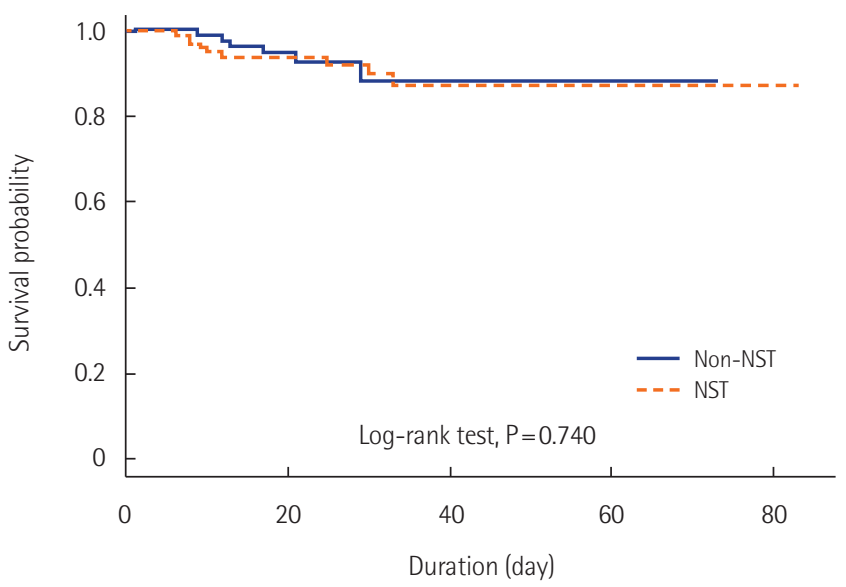

Figure 2. Comparison of survival between patients admitted to the trauma intensive care unit in the nutrition support team (NST) group and non-NST group.

Table 4. Mortality according to NST treatment

\begin{tabular}{lcc}
\hline \multirow{2}{*}{ Variable } & \multicolumn{2}{c}{ Overall mortality } \\
\cline { 2 - 3 } & $\mathrm{HR}(95 \% \mathrm{Cl})$ & P-value \\
\hline Crude model & $1.19(0.42-3.40)$ & 0.740 \\
Adjusted model & $2.11(0.66-6.78)$ & 0.211 \\
\hline
\end{tabular}

Crude model: adjusted for age and sex; Adjusted model: adjusted for age, sex, major shock, ISS, and APACHE II.

NST: nutrition support team; HR: hazard ratio; $\mathrm{Cl}$ : confidence interval; ISS: injury severity score; APACHE: Acute Physiology and Chronic Health Evaluation.

Table 3. Nutritional and clinical outcomes according to NST consultation after propensity score matching

\begin{tabular}{lccc}
\hline Variable & Non-NST $(n=102)$ & NST $(n=102)$ & P-value \\
\hline Total delivered/required caloric ratio in TICU (\%) & $66.7 \pm 37.6$ & $80.4 \pm 30.1$ & 0.007 \\
Total delivered/required protein ratio in TICU (\%) & $68.3 \pm 54.7$ & $93.1 \pm 36.0$ & $44(43.1)$ \\
Adequate calorie supply in TICU & $28(31.8)$ & $49(48.0)$ & 0.001 \\
Adequate protein supply in TICU & $23(25.8)$ & $8.7 \pm 10.3$ & 0.002 \\
Duration of TICU admission (day) & $6.6 \pm 5.5$ & $29.4 \pm 19.8$ & 0.074 \\
Duration of hospitalization (day) & $23.4 \pm 15.9$ & $8(7.8)$ & 0.018 \\
ICU mortality & $5(4.9)$ & $9(8.8)$ & 0.390 \\
Overall mortality & $6(5.9)$ & 0.421 & \\
\hline
\end{tabular}

Values are presented as mean \pm standard deviation or number (\%).

NST: nutrition support team; TICU: trauma intensive care unit; ICU: intensive care unit. 
rounds more than twice a week with the current reimbursement plan for the NST program. Therefore, it is difficult to continuously check nutrition status, and this appears to have failed to improve clinical outcomes since continuous and detailed nutrition support was difficult.

EN is desired over PN in trauma patients, and it is associated with decreased infections, decreased mechanical ventilation days, and a shorter length of ICU stay [13-16]. However, the results of the present study showed that $82.3 \%$ of patients initially received PN and nutrition was initiated 2.4 days after admission on average. This is very different to the results of a recent large, multi-national, multi-institutional study, in which $81 \%$ of trauma patients received EN [11]. In Korea, PN is used in a high proportion of surgical patients, and PN is commonly used in patients receiving EN [17]. Reimbursement provided for the NST program by the NHI began on a large scale in 2015 to address these nutritional problems [6]. However, Korean physicians' perceptions of PN complications in trauma patients are inconsistent, and a consensus for active use of EN has not been reached [18]. As a result, the change in practice appears to be occurring slowly. In our study, approximately $50 \%$ of patients underwent surgery during their TICU admission. Preoperative midnight fasting was requested by anesthesiologists for most surgeries, except for emergency surgery before TICU admission. Recent recommendations are that patients can consume a clear liquid diet up until 2 hours before elective surgery in order to minimize perioperative fasting. However, anesthesia has not yet been performed following this policy in the hospital used in our study $[19,20]$. For this reason, it is thought that patients were initiated on PN, instead of EN, because there was a possibility that they would require an operation during their TICU stay.

Reimbursement for the NST program in Korea, which began in 2015, has had a positive impact on nutritional practice, but it has not completely addressed fundamental nutrition problems [6]. In addition, multidisciplinary rounds are only performed twice a week [6]. As the physicians in charge have a limited understanding of nutrition therapy, compliance with recommendations given by the NST is also low [18]. In order to solve these problems, it is necessary to ensure continuity in nutrition support for patients by organizing the NST round with the multidisciplinary round in the ICU. It is also important to educate physicians, nurses, pharmacists, and nutritionists who manage critically ill patients and to promote NSTs [21].

This study has some limitations. First, it does not show the overall outcome of NST operation in all institutions, because it was performed at a single institution. Second, it was difficult to include information regarding complications, and there was also selection bias between the NST group and the nonNST group, because this was a retrospective study. Third, it is difficult to know whether the recommendations were reflected in the patient's actual management, since this study did not include information about compliance with NST recommendations by the physicians in charge. Despite these limitations, the results of this study showed the current state of nutrition support in a Korean TICU and provided meaningful information on the effects of the NST project by the NHI on outcomes in trauma patients.

In conclusion, a multidisciplinary team approach for nutrition support significantly increased the nutritional intake of TICU patients. However, additional prospective studies with actual nutrition support or other factors affecting clinical outcomes are needed in order to confirm the impact of NST on clinical outcomes.

\section{CONFLICT OF INTEREST}

No potential conflict of interest relevant to this article was reported.

\section{ORCID}

Eunsuk Oh https://orcid.org/0000-0001-5106-0781

Hongjin Shim https://orcid.org/0000-0003-3073-3806

Hyon Ju Yon https://orcid.org/0000-0002-0376-1994

Jin Sil Moon https://orcid.org/0000-0002-6197-7742

Dae Ryong Kang https://orcid.org/0000-0002-8792-9730

Ji Young Jang https://orcid.org/0000-0001-6372-4194

\section{AUTHOR CONTRIBUTIONS}

Conceptualization: EO, HS, JYJ. Data curation: EO, HJY, JYJ. Formal analysis: JSM, DRK, JYJ. Methodology: EO, HS, DRK, JYJ. Project administration: HS, JYJ. Visualization: JSM, JYJ. Writing-original draft: EO, JYJ. Writing-review \& editing: all authors.

\section{REFERENCES}

1. Edington J, Boorman J, Durrant ER, Perkins A, Giffin CV, James $\mathrm{R}$, et al. Prevalence of malnutrition on admission to four hospitals in England. The Malnutrition Prevalence Group. Clin Nutr 2000;19:191-5.

2. Bruun LI, Bosaeus I, Bergstad I, Nygaard K. Prevalence of mal- 
nutrition in surgical patients: evaluation of nutritional support and documentation. Clin Nutr 1999;18:141-7.

3. Correia MI, Waitzberg DL. The impact of malnutrition on morbidity, mortality, length of hospital stay and costs evaluated through a multivariate model analysis. Clin Nutr 2003;22:235-9.

4. Mogensen KM, Robinson MK, Casey JD, Gunasekera NS, Moromizato T, Rawn JD, et al. Nutritional Status and mortality in the critically ill. Crit Care Med 2015;43:2605-15.

5. Alberda C, Gramlich L, Jones N, Jeejeebhoy K, Day AG, Dhaliwal $\mathrm{R}$, et al. The relationship between nutritional intake and clinical outcomes in critically ill patients: results of an international multicenter observational study. Intensive Care Med 2009;35:1728-37.

6. Lee JS, Kang JE, Park SH, Jin HK, Jang SM, Kim SA, et al. Nutrition and clinical outcomes of nutrition support in multidisciplinary team for critically ill patients. Nutr Clin Pract 2018; 33:633-9.

7. Doig GS, Heighes PT, Simpson F, Sweetman EA. Early enteral nutrition reduces mortality in trauma patients requiring intensive care: a meta-analysis of randomised controlled trials. Injury 2011;42:50-6.

8. Dissanaike S, Pham T, Shalhub S, Warner K, Hennessy L, Moore EE, et al. Effect of immediate enteral feeding on trauma patients with an open abdomen: protection from nosocomial infections. J Am Coll Surg 2008;207:690-7.

9. Wang X, Dong Y, Han X, Qi XQ, Huang CG, Hou LJ. Nutritional support for patients sustaining traumatic brain injury: a systematic review and meta-analysis of prospective studies. PLoS One 2013;8:e58838.

10. Wasiak J, Cleland H, Jeffery R. Early versus delayed enteral nutrition support for burn injuries. Cochrane Database Syst Rev 2006;(3):CD005489.

11. Wade CE, Kozar RA, Dyer CB, Bulger EM, Mourtzakis M, Heyland DK. Evaluation of nutrition deficits in adult and elderly trauma patients. JPEN J Parenter Enteral Nutr 2015;39:449-55.

12. Jang JY, Shim H, Kwon HY, Chung H, Jung PY, Kim S, et al. Improvement of outcomes in patients with pelvic fractures and hemodynamic instability after the establishment of a Korean regional trauma center. Eur J Trauma Emerg Surg 2019;45:107-
13.

13. Kudsk KA, Croce MA, Fabian TC, Minard G, Tolley EA, Poret $\mathrm{HA}$, et al. Enteral versus parenteral feeding. Effects on septic morbidity after blunt and penetrating abdominal trauma. Ann Surg 1992;215:503-11.

14. Moore FA, Feliciano DV, Andrassy RJ, McArdle AH, Booth FV, Morgenstein-Wagner TB, et al. Early enteral feeding, compared with parenteral, reduces postoperative septic complications: the results of a meta-analysis. Ann Surg 1992;216:17283.

15. Rhee P, Hadjizacharia P, Trankiem C, Chan L, Salim A, Brown $\mathrm{C}$, et al. What happened to total parenteral nutrition? The disappearance of its use in a trauma intensive care unit. J Trauma 2007;63:1215-22.

16. Plurad D, Green D, Inaba K, Belzberg H, Demetriades D, Rhee P. A 6-year review of total parenteral nutrition use and association with late-onset acute respiratory distress syndrome among ventilated trauma victims. Injury 2009;40:511-5.

17. Lee SH, Jang JY, Kim HW, Jung MJ, Lee JG. Effects of early enteral nutrition on patients after emergency gastrointestinal surgery: a propensity score matching analysis. Medicine (Baltimore) 2014;93:e323.

18. Jeong HS, Teong CH, Choi, YJ, Kim WJ, Lee AR. Attitudes of medical staff and factors related to nutritional support for patient care in a university hospital. J Clin Nutr 2014;6:37-41.

19. Practice guidelines for preoperative fasting and the use of pharmacologic agents to reduce the risk of pulmonary aspiration: application to healthy patients undergoing elective procedures: an updated report by the American Society of Anesthesiologists task force on preoperative fasting and the use of pharmacologic agents to reduce the risk of pulmonary aspiration. Anesthesiology. 2017;126:376-93.

20. Smith I, Kranke P, Murat I, Smith A, O'Sullivan G, Søreide E, et al. Perioperative fasting in adults and children: guidelines from the European Society of Anaesthesiology. Eur J Anaesthesiol 2011;28:556-69.

21. DeChicco R, Neal T, Guardino JM. Developing an education program for nutrition support teams. Nutr Clin Pract 2010; 25:481-9. 